\title{
Black Community College Students and Opinions and Beliefs on Weight in the Community: A Focus Group Study
}

\author{
Janna Stephens ${ }^{1 *}$, Antoinette Perkins ${ }^{2}$ \\ ${ }^{1}$ Ohio State University College of Nursing, Columbus, USA \\ ${ }^{2}$ Sports \& Exercise Studies, Columbus State Community College, Columbus, USA \\ Email: ^Stephens.653@osu.edu, Aperki01@cscc.edu
}

How to cite this paper: Stephens, J. and Perkins, A. (2019) Black Community College Students and Opinions and Beliefs on Weight in the Community: A Focus Group Study. Health, 11, 57-66.

https://doi.org/10.4236/health.2019.111007

Received: December 6, 2018

Accepted: January 14, 2019

Published: January 17, 2019

Copyright $\odot 2019$ by author(s) and Scientific Research Publishing Inc. This work is licensed under the Creative Commons Attribution International License (CC BY 4.0).

http://creativecommons.org/licenses/by/4.0/

\section{c) (i) Open Access}

\begin{abstract}
Objective: The purpose of this study was to better understand black community college students' beliefs on obesity in their community. Methods: Participants were 18 - 21 years old and African American/Hispanic Black. Focus group sessions were conducted with a discussion guide. Thematic analysis was used to analyze results. Results: Five focus groups were conducted. Four major themes emerged: My Culture Matters, The Effects of Slavery are Still Present, Meet Me Where I Want to Be, Not Where Society Wants Me, and If They Have Been Fat, They Get Me. It is clear that weight programs need to be sensitive to cultural preferences. Conclusion: Our results present unique factors related to overweight/obesity in the Black community.
\end{abstract}

\section{Keywords}

Overweight, Focus Groups, Young Adult, Minority Groups

\section{Introduction}

Overweight and obesity represent a large public health concern in the United States and can lead to adverse health outcomes, including diabetes, sleep apnea, some forms of cancer, and heart disease [1] [2] [3] [4]. Young adults (all races) have high overweight and obesity rates, with $60.3 \%$ having a body mass index (BMI) $\geq 25$ [4]. Rates of overweight and obesity in African American and Hispanic (Black and White) young adults are higher than the national average with $71.9 \%$ and $71.7 \%$ being overweight or obese respectively [4]. Young adults may experience significant weight gain during the transition years into college, where adolescents become independent young adults who are developing unhealthy 
eating and sedentary lifestyle habits [5]-[10]. Considering the significant health disparity for African American and Hispanic young adults, it is important to reach these populations during these critical years when lifelong habits are being formed.

Although it is well established that college is a time when individuals tend to gain weight, it is unclear what additional unique factors may be related to weight gain in the African American and Black population and it remains unclear their perspective on specific factors that may lead to overweight and obesity. It is known through studies mostly conducted in Caucasian students that poor nutrition and lack of physical activity are potential causes of overweight/obesity in all populations [6] [11] [12] [13]. However, the literature has established that there are additional factors that can potentially lead to unhealthy habits, such as cultural beliefs related to exercise, cultural values on body image, cultural patterns of nutrition, neighborhood discrimination with regards to healthy food advertisement, and other factors [14] [15].

The purpose of this qualitative focus group study was to better understand young adult black community college students' opinions and beliefs on obesity in their community.

\section{Methods}

This study used a qualitative exploratory design with focus group sessions. The study was approved by the Columbus State Community College (CCSC) Institutional Review Board (IRB) and the Ohio State University IRB.

CSCC is a community college located in large urban community with over 30,000 students and $40 \%$ minority. Young adults were recruited through posters and flyers hung in and around the CSCC campus. In addition, announcements were made in several classrooms to inform students of the current study. Word of mouth was another popular method of recruitment. In order to participate, individuals had to be between 18 and 21 years of age, a current CSCC student, and they had to identify as African American or Hispanic. There were no requirements based on body mass index of the participant and this information was not collected. Students all over campus were encouraged to join the study and the authors attempted to engage all types of students who fit criteria.

This study was guided by phenomenology whereas the goal was to describe the experience/perspective of overweight/obesity in the Black community. Although participants were not required to be overweight or obese to participate, the focus group sessions were focused on both the individual and family experience of obesity and how obesity may impact at various levels. Focus groups were selected as the appropriate method for data collection due to a lack of time for students to complete one to one interviews and for ease of recruitment purposes into the study.

Five focus group sessions were conducted between February 2016 and April 2016. Sample size was determined once saturation was met. A single moderator, who identified as African American (age > 40), conducted all five sessions with a 
single assistant who identified as White (age $<30$ ) attending all sessions to record notes on body language, facial expressions, and other environmental situations that the tape recorders would not capture. The moderator used a discussion guide to ensure all sessions were assessing the same questions regarding general thoughts on overweight/obesity and weight loss interventions (see Table 1). All sessions were conducted in a casual environment in a private classroom on the CSCC campus. Each session lasted between 40 and 60 minutes. The moderator read the consent form and each individual gave written informed consent prior to starting the focus group session. All sessions were open to both genders.

All sessions were tape-recorded with participant's knowledge of the recordings. Three handheld tape recorders were placed around the room in various places to capture all dialogue occurring during the session. The moderator and assistant introduced themselves and discussed the purpose of the study with the group and the rules for the focus group session. The moderator emphasized that all responses and discussion were welcomed and that no response was considered correct or incorrect. Participants were reminded to be courteous to the responses of others and to respect all opinions in the room. They were also reminded that they did not have to answer any question that made them uncomfortable or that they did not feel like discussing. The discussion guide was then used to ask open-ended questions to the group as well as asking minimal directive questions. Participants that remained quiet were periodically asked their opinion in order to engage them in conversation. Following the sessions, the moderator and assistant met immediately to discuss and debrief on the sessions and the notes taken during the session.

Qualitative analysis of each focus group session was done using a process of thematic analysis, like in our previous studies [10]. The process began with an initial read of the transcribed data and reading of the notes taken during the session by the primary investigator. The analysis procedure consisted of multiple readings of the transcripts to identify key words or phrases. Hand coding was used as to follow a phenomenology approach to "live with the data". The coding began during the second read of the data. The relevant passages were coded

Table 1. Example discussion guide questions.

\begin{tabular}{cll}
\hline Topic & \multicolumn{1}{c}{ Opening Question } & \multicolumn{1}{c}{ Follow-Up Question } \\
\hline $\begin{array}{c}\text { General } \\
\text { perspectives } \\
\text { on weight }\end{array}$ & $\begin{array}{l}\text { I would like to hear your thoughts on } \\
\text { weight and weight gain during college. } \\
\text { What are your thoughts and what } \\
\text { do you see across campus? }\end{array}$ & \\
$\begin{array}{c}\text { General } \\
\text { perspectives } \\
\text { on weight }\end{array}$ & $\begin{array}{l}\text { How do you think race } \\
\text { plays a role in weight } \\
\text { and weight gain during college? }\end{array}$ & $\begin{array}{l}\text { Could you explain if you think } \\
\text { African Americans or Blacks have } \\
\text { than other races? }\end{array}$ \\
height loss & $\begin{array}{l}\text { Can you talk about what you would } \\
\text { look for in a health coach } \\
\text { interventions }\end{array}$ & $\begin{array}{l}\text { Would you want someone who is } \\
\text { close to your age? How would you } \\
\text { feel if this person was a healthcare } \\
\end{array}$
\end{tabular}


initially using words from the participants themselves. Subsequent readings of the transcripts and the passages coded in similar ways yielded categories of data describing the critical issues for the participants. Categories from each topic were combined to form themes, which provided a comprehensive view of the data.

\section{Results}

A total of five focus groups were conducted with 28 participants ( $46 \%$ male). All participants identified as African American or Black and were community college students. Data was not collected on ethnicity of participants. The results across focus groups are organized by major themes with a general explanation of that theme with exemplar quotes. Overall, there were four major themes that emerged from the focus group sessions and the results are organized by theme.

\section{My Culture Matters}

The participants across all groups stressed that minority cultures, specifically African American and Black Hispanics, are very different from their Caucasian counterparts and that this plays a significant role in weight gain and the ability to lose weight. One particular factor that emerged was that African Americans, in general, have more going on in their lives.

Female 1: "So when you factor it all in, it's just that, you know? African-Americans kinda have more to deal with."

Participants stressed that African Americans, particularly women, tend to have more responsibilities than their Caucasian counterparts. The African American women attending community college are likely working at least one job, perhaps raising children or siblings, possibly head of household, and have other life duties. Also, the women felt that they have to try harder to be liked and seen as important in society.

Female 2: "That's the beauty in it, but in order to get there, I feel like as, you know, black African women we have to almost go through twice as much to just get to love, to just be like, yeah, you don't look bad, girl."

In addition, the idea of diet related to culture emerged as a contributor to obesity in the United States (US). Participants stressed the use of seasonings in their food, massive amounts of salt in the diet, and lack of activity in the US compared to other countries of origin.

Male 1: "Um, and I reckon people, what I see in those kids, um, more often than not, you see a lot more obesity because, uh, back home, like a diet, back in Puerto Rico we eat a lot of rice, lot of beans, um, lots of carbs."

Male 2: "Over here a lot of times they keep that same diet but they're not as active. They' re driving, taking a bus, um, cos everything's so much further away. Um, so you see a lot of weight gain in those communities because of that. Also, um, I don't know, a-, Americans have really, really crappy diets I think, um."

In addition, although culture matters, it was interesting to hear that race and ethnicity do not matter to the majority of participants when asked about characteristics they would prefer in a health coach. Participants stressed that they were 
not concerned with learning from someone who is the same race/ethnicity as them, in fact some participants preferred a "white" health coach. But, participants, particularly African American women, stressed that they want someone who can understand the unique differences of their culture. These differences ranged from the use of certain body powders, hair perms, exercise/sweating perception, cultural foods, and more.

Female 3: "So I would really like a cumulative lifestyle when it comes to skin and hair maintenance as well."

Female 4: "Um, even when I use my hair, Id use curry leaves in my hair oil because it's an Indian technique that involves like just maintaining the growth and stuff. So there are a lot of things that, um, really could cater towards black women or just of African descent or whatever the case may be."

The Effects of Slavery Are Still Present

Across the focus groups there was rich discussion about the long-lasting effects of slavery in the United States. The participants stressed that the diet consumed by slaves was full of fat and sodium and that those particular taste preferences stuck with the culture.

Male 1: "Um, for a number of reasons. Uh, firstly especially within African-American communities, um, not, not tryna pull like a race card, but like, basically back in the day obviously, you know, like slaves ate what they were fed, you know, the shit piles, the food, you know, that nobody wanted to eat, and that has obviously become part of the culture. You eat soul food, be it fried stuff, and it $s$ delicious, don't get me wrong."

The discussion also focused on the idea that healthier slaves were those who had the ability to hold more weight. Those slaves survived, thus leading to a line of heavier African American individuals.

Female 5: "You know, who came through the slave trade. You know, a lot of the slaves who were smaller died on the way over, so the ones who were able to retain more of the salt and stay bigger."

Male 3: "You know, being in African with scarce food, biologically you're gonna retain more fat in your body. That's why black females have usually huge butts and huge breasts."

The everlasting effects of slavery are also motivations for weight loss. Participants recognized the conditions associated with overweight and obesity and are actively trying to prevent those conditions in their lives. The understanding is that it is an uphill battle due to genetics.

Male 4: " $P m$ just standing, like I have a shit ton of diabetes in my family, a whole bunch of heart problems. My family are like, $I m$ already aware of these things and I m like trying, you know, avoid those things."

Male 5: "Exactly, culturally. You know what I mean, like black guys like the woman thicker so female are oftentimes thicker than-I mean, genetically they' re already thicker."

Meet Me Where I Want to Be, Not Where Society Wants Me

The idea of becoming what the "general society" sees as beautiful is not the 
goal of most participants. Overall, participants, especially females, were not interested in being what they considered "skinny." Participants believed that most diet and exercise programs would gear them towards being a particular size (size 2 was a common thought) or a particular shape (no curves).

Female 6: "As for me, I think nothing influences me. I just wanna be me. I just wanna be healthy."

Female 7: "To really understand and love yourself, and when you say, you know. These hips come from my home country. These, these lips come from this or my skin comes from this, I love it, like it's not what makes me, um, you, you know, I shouldn't cower in fear because of my traits. I should, you know, love my traits. That's what makes me, me. That's what connects me to my sisters and I. That's what connects me to my hair. That's why I m looking at your hair like your hair is gorgeous."

Overall, participants preferred to join a program that allowed them to select their goals and would not force them to lose a certain amount of weight. In addition, women in particular want to be respected and not judged for choosing to be whatever weight they are comfortable being.

If They Have Been Fat, They Get Me

When asked what type of health coach participants would prefer, the majority stated they think previous struggle with weight would be beneficial. There was an overwhelming feeling of desiring a health coach who had "walked in their shoes" or "was overweight in the past." Participants felt they could only learn from someone who would understand the difficulties of starting a workout regime or attempting to lose weight.

Female 8: "That's not like a, yeah, that's not like a, if he had never been fat before, I d be like, you don't know my struggle."

Male 6: But if you have a coach that could tell you, like you say, "You don't know what I go through," and he's talking about, "Yes, I do. I used to be just like you, maybe even worse, but look at me now."

Male 7: "Cos they kind of know. It's like, b-, they know, like, let me say... They know the struggle. The struggles and then how it feels."

In addition, learning from someone who understands overweight/obesity would allow the participants to go at their own pace. Learning from someone who is well beyond their own abilities is not attractive to the participants.

Female: "You know like, uh, there was a workout online, there was a, a guy, a male ballet dancer. He's probably been dancing since he was like two, and he's, like even the work, the girls in the back that were working out with him couldn't keep up with him, see, and that's what I don't want. I don't want anybody that's like, you' re working out at your level."

In addition to the themes above, the participants were overwhelmingly excited about the possibility to participate in a program to increase healthy eating and exercise, even those who identified through discussion that they are currently a normal weight. The idea of using a Smartphone application and working with a health coach was received positively. 


\section{Discussion}

Our findings suggest interesting areas for improvement in how we create and individualize weight programs geared toward minority young adults. It has been understood for years that being sensitive to one's culture is imperative in research. Data suggests that lower socioeconomic status, family history, having children, and poorer health status are correlates of African American students' high body mass index [16]. Our data suggests that the students are aware that their additional responsibilities, such as having children, may create an environment where it is more difficult to lose weight or maintain a healthy eating and exercise regimen. They are also aware of poor family health history, such as diabetes and heart disease, but rarely did they discuss low socioeconomic status as being related to unhealthy habits.

When examining data to support the participant beliefs on slavery, we found that African American women with slave ancestry tend to give birth to babies who weigh less than white counterparts [17]. The data suggest that poor nutrition status during slavery led to poor nutritional status of children and adults during slavery [17]. These patterns, today, have led to Black women giving birth in the United States to lower birth weight babies. Therefore, there seems to be a difference between beliefs of African American young adults in our study and the scientific data presented. It is possible that the exploration of our participant beliefs have yet to be explored due to the complicated nature of that type of study. There is support that slaves were fed diets high in fat and starch, as our participants discuss [18]. These diets were not sufficient and led to slaves, sometimes, stealing food from their master etc. Perhaps the connection is more based that slaves were given small rations and therefore, overtime, Blacks in the United States learned to eat more from times when they had less. More exploration is needed on this topic.

Similar studies report family as influential role models in terms of dieting and exercise. One study by D'Alonzo and Fischetti, reported that African Americans looked at family members to influence them to exercise [19]. Combined with our data where family members were seen as motivations, both positive and negative (don't want diabetes), it might be important to include the family unit in an intervention targeted toward minority young adults.

When examining our data that participants want a health coach that has experienced weight loss, we found additional data to support this notion. A study examining minority college students found that Blacks and Hispanics look for people who look like them when they search for a place to exercise. They also discuss joining an exercise program at their "level" which is similar to what our participants expressed.

Our results reflect previous studies examining the ideal body image in minority women. In a study conducted by Franko et al. (2012), they found that Latina college students experienced confusion between the ideal body image portrayed by whites and the ideal portrayed by Latinas [20]. In addition, the Latina women 
felt they were born with curves, hinting at the same idea that genetics plays a major role when examining minority cultures and weight. The women also expressed the same concerns over becoming a certain size and the ideals behind "being a size 2". This data supports that minority women need to be approached with an open-mindedness surrounding ideal body image and that health coaches or other individuals should allow the participant to explore what body size/shape is ideal for them. In addition, two similar studies in African American females found that they also, like our participants, want to define their own ideals and be themselves, not be forced into what society believes as beautiful [21] [22]. Therefore, it is key that we develop programs that allow the participant to develop and set their own goals. Weight loss should not necessarily be the primary objective for these individuals. Perhaps a focus on additional physical activity and increasing healthy eating would be more beneficial, with weight loss being a secondary outcome if necessary.

Limitations of this study include: a single researcher conducting data analysis, homogeneous responses across focus groups, inability to identify participants by characteristics outside of male/female, and homogeneous population due to recruitment at a single location.

To our knowledge, this is the first focus group study to examine and discover new factors that minority community college students believe to be related to overweight and obesity. We discovered data that is congruent with other current studies, but also discovered new information that can and should be used to build interventions for weight loss in minority young adults. One important new factor was the want for a health coach to have experience with losing weight or being overweight and they do not need to be of the same racial group.

\section{Conclusion}

Across all five focus groups, the overwhelming majority of students believe that slavery plays a large role in why African Americans are disproportionately burdened with overweight and obesity today in the United States. In addition, this study sheds new light on what minority students look for in a health coach. Moving forward, studies can focus on hiring health coaches that have suffered from overweight/obesity and that understand cultural differences in terms of personal hygiene, exercise preferences, and more.

\section{Funding}

Funding is provided by Ohio State University College of Nursing.

\section{Conflicts of Interest}

No conflicts of interest.

\section{References}

[1] Flegal, K., Graubard, B., Williamson, D. and Gail, M. (2005) Excess Deaths Asso- 
ciated with Underweight, Overweight, and Obesity. JAMA, 293, 1861-1867. https://doi.org/10.1001/jama.293.15.1861

[2] Gortmaker, S., Must, A., Perrin, J., Sobol, A. and Dietz, W. (1993) Social and Economic Consequences of Overweight in Adolescence and Young Adulthood. New England Journal of Medicine, 329, 1008-1012. https://doi.org/10.1056/NEJM199309303291406

[3] Poirier, P., Giles, T., Bray, G., et al., American Heart Association, Obesity Committee of the Council on Nutrition, Physical Activity, and Metabolism (2006) Obesity and Cardiovascular Disease: Pathophysiology, Evaluation, and Effect of Weight Loss: An Update of the 1997 American Heart Association Scientific Statement on Obesity and Heart Disease from the Obesity Committee of the Council on Nutrition, Physical Activity, and Metabolism. Circulation, 113, 898-918.

https://doi.org/10.1161/CIRCULATIONAHA.106.171016

[4] Ogden, C., Carroll, M., Kit, B. and Flegal, K. (2014) Prevalence of Childhood and Adult Obesity in the United States, 2011-2012. JAMA, 311, 806-814.

https://doi.org/10.1001/jama.2014.732

[5] Desai, M., Miller, W., Staples, B. and Bravender, T. (2008) Risk Factors Associated with Overweight and Obesity in College Students. Journal of American College Health, 57, 109-114. https://doi.org/10.3200/JACH.57.1.109-114

[6] Greaney, M., Less, F., White, A., et al. (2009) College Students' Barriers and eNablers for Healthful Weight Management: A Qualitative Study. Journal of Nutrition Education and Behavior, 41, 281-286. https://doi.org/10.1016/j.jneb.2008.04.354

[7] Gropper, S., Simmons, K., Connell, L., et al. (2012) Weight and Body Composition Changes during the First Three Years of College. Journal of Obesity, 2012, Article ID: 634048. https://doi.org/10.1155/2012/634048

[8] Lacaille, L., Dauner, K., Krambeer, R. and Pederson, J. (2011) Psychosocial and Environmental Determinants of Eating Behaviors, Physical Activity, and Weight Change among College Students: A Qualitative Analysis. Journal of American College Health, 59, 531-538. https://doi.org/10.1080/07448481.2010.523855

[9] Smith-Jackson, T. and Reel, J. (2012) Freshman Women and the "Freshman 15": Perspectives on Prevalence and Causes of College Weight Gain. Journal of American College Health, 60, 14-20. https://doi.org/10.1080/07448481.2011.555931

[10] Stephens, J., Moscou-Jackson, G. and Allen, J. (2015) Young Adults, Technology, and Weight Loss: A Focus Group Study. Journal of Obesity, 2015, Article ID: 379769. https://doi.org/10.1155/2015/379769

[11] American College Health Association (2015) National College Health Assessment II: Undergraduate Student Reference Group Executive Summary.

[12] Anderson, D., Shapiro, J. and Lundgren, J. (2003) The Freshman Year of College as a Critical Period for Weight Gain: An Initial Evaluation. Eating Behaviors, 4, 363-367. https://doi.org/10.1016/S1471-0153(03)00030-8

[13] Fedewa, M., Das, B., Evans, E. and Dishman, R. (2014) Change in Weight and Adiposity in College Students: A Systematic Review and Meta-Analysis. American Journal of Preventive Medicine, 47, 641-652. https://doi.org/10.1016/j.amepre.2014.07.035

[14] Capodilupo, C. and Kim, S. (2014) Gender and Race Matter: The Importance of Considering Intersections in Black Women's Body Image. Journal of Counseling Psychology, 61, 37-49. https://doi.org/10.1037/a0034597

[15] Kwarteng, J., Schulz, A., Mentz, G., Israel, B., Shanks, T. and Perkins, D. (2016) Neighborhood Poverty. Perceived Discrimination and Central Adiposity in the 
USA: Independent Associations in a Repeated Measures Analysis. Journal of Biosocial Science, 48, 709-722. https://doi.org/10.1017/S0021932016000225

[16] Gary, T., Gross, S., Browne, D. and LaVeist, T. (2006) The College Health and Wellness Study: Baseline Correlates of Overweight among African Americans. Journal of Urban Health, 83, 253-265. https://doi.org/10.1007/s11524-005-9026-1

[17] Jasienska, G. (2009) Low Birth Weight of Contemporary African Americans: An Intergenerational End of Slavery? American Journal of Human Biology, 21, 24. https://doi.org/10.1002/ajhb.20824

[18] Educational Broadcasting Corporation (2004) Slavery and the Making of America.

[19] D'alonzo, K. and Fischetti, N. (2008) Cultural Beliefs and Attitudes of Black and Hispanic College-Age Women toward Exercise. Journal of Transcultural Nursing, 19, 175-183. https://doi.org/10.1177/1043659607313074

[20] Franko, D., Coena, E., Roehriga, J., et al. (2012) Considering J.Lo and Ugly Betty: A Qualitative Examination of Risk Factors and Prevention Targets for Body Dissatisfaction, Eating Disorders, and Obesity in Young Latina Women. Body Image, 9, 381-387. https://doi.org/10.1016/j.bodyim.2012.04.003

[21] Webb, O.J., Benjamin, C.C., Gammon, C., McKee, H.C. and Biddle, S.J.H. (2013) Physical Activity, Sedentary Behaviour and Physical Self-Perceptions in Adolescent Girls: A Mediation Analysis. Mental Health and Physical Activity, 6, 24-29. https://doi.org/10.1016/j.mhpa.2012.08.005

[22] Webb, J., Warren-Findlowb, J., Choua, Y. and Adams, L. (2013) Do You See What I See? An Exploration of Inter-Ethnic Ideal Body Size Comparisons among College Women. Body Image, 10, 369-379. https://doi.org/10.1016/j.bodyim.2013.03.005 